\title{
Differential Contributions of Conformation Extension and Domain Unfolding to Properties of Fibronectin Nanotextiles
}

\section{Citation}

Deravi, Leila F., Tianxiang Su, Jeffrey A. Paten, Jeffrey W. Ruberti, Katia Bertoldi, and Kevin Kit Parker. 2012. Differential contributions of conformation extension and domain unfolding to properties of fibronectin nanotextiles. Nano Letters 12(11): 5587-5592.

\section{Published Version}

doi: $10.1021 / \mathrm{nl} 302643 \mathrm{~g}$

\section{Permanent link}

http://nrs.harvard.edu/urn-3:HUL.InstRepos:10919789

\section{Terms of Use}

This article was downloaded from Harvard University's DASH repository, and is made available under the terms and conditions applicable to Open Access Policy Articles, as set forth at http:// nrs.harvard.edu/urn-3:HUL.InstRepos:dash.current.terms-of-use\#OAP

\section{Share Your Story}

The Harvard community has made this article openly available.

Please share how this access benefits you. Submit a story.

\section{Accessibility}




\section{Differential Contributions of Conformation}

\section{Extension and Domain Unfolding to Properties of}

\section{Fibronectin Nanotextiles}

Leila F. Deravi ${ }^{a}$, Tianxiang Su ${ }^{b}$, Jeffrey A. Paten ${ }^{c}$, Jeffrey W. Ruberti ${ }^{c}$, Katia Bertoldi ${ }^{b}$, Kevin Kit Parker $^{a, *}$

${ }^{\mathrm{a}}$ Disease Biophysics Group, Wyss Institute for Biologically Inspired Engineering, School of Engineering and Applied Sciences, Harvard University, Cambridge, MA 02138

${ }^{\mathrm{b}}$ School of Engineering and Applied Sciences, Harvard University, Cambridge, MA 02138

${ }^{c}$ Department of Mechanical and Industrial Engineering, Northeastern University, Boston, MA 02139

KEYWORDS: multimodular proteins, fibrillogenesis, surface assembly, matrix elasticity, plastic deformation 
Fibronectin (FN) textiles are built as nanometer thick fabrics. When uniaxially loaded, these fabrics exhibit a distinct threshold between elastic and plastic deformation with increasing stretch. Fabric mechanics are modeled using an eight-chain network and two-state model, revealing that elastic properties of FN depend on conformational extension of the protein and that plastic deformation depends on domain unfolding. Our results suggest how the molecular architecture of a molecule can be exploited for designer mechanical properties of a bulk material. 
Cells build, sculpt, and maintain the extracellular protein networks that underlie the structural integrity of a tissue. ${ }^{1-7}$ The adaptability and functionality of the extracellular matrix (ECM) is due, in part to, the hierarchal architecture of its constituent protein fibers. The primary structure of extracellular matrix proteins dictates its secondary and tertiary structure folding, which endows the ECM network with unique chemical and mechanical properties. ${ }^{8-9}$ The hierarchical organization of the ECM can be recapitulated in synthetic protein based materials for applications requiring robust chemical and mechanical functionality over a broad range of length scales. Thus, an important detail in the design and assembly of biomimetic protein textiles is an understanding of how the vertically-integrated structures of the protein fibril behave under mechanical loading.

Some ECM proteins offer certain advantages for manufacturing and endowing protein textiles with unique functionalities. For example, Fibronectin (FN) is a $450 \mathrm{kDa}$ dimer composed of modular folded domains (I, II, or III) that are categorized by their sequence homology. ${ }^{10-14}$ Unlike the FN I and II domains, the $\beta$-sheet rich, FN III domains do not contain internal disulfide bonds - a characteristic that enables the large extensibility of FN in the presence of tensile load. FN undergoes conformational changes from a soluble, compact state to an extended conformation under cell generated strain. ${ }^{1,6,10-11,13,15}$ In the extended conformation, additional FN molecules bind at FN I domains, polymerizing to form an insoluble protein network..$^{10-11}$ While it is understood that cell coupling and cytoskeletal contraction induce conformational changes required for fibrillogenesis in the extracellular space, how FN fibers respond to strain is debated. Two structural models have been proposed to describe the mechanical properties of FN fibers. In the first model, elasticity within FN fibers is due to their conformational extension without domain unfolding. ${ }^{10,16}$ When FN fibers are relaxed, constituent FN molecules are in a 
compact form. Under tensile load, the fibers stretch due to conformational extension. In the second model, relaxed FN matrix fibers contain polymerized FN that is already in an extended conformation, where elasticity is due to individual FN III domains that unfold and refold within the fibril. ${ }^{17-18}$

We reasoned FN fibers would display different and distinguishable mechanical properties during conformational and domain unfolding. To test our hypothesis, we manufactured FN fabrics by a previously published method that requires assembly of protein fabrics on a surface with microcontact printing and then its subsequent release. ${ }^{19}$ This process is illustrated in Figure 1a; a schematic representation of the experiment is shown in Supporting Information Figure S1a, where a micropatterned FN ribbon is released from a PIPAAm substrate. When released, the ribbons contract, curling along their transverse axis to form nanoFabrics (Supporting Information Figure S1a-d,). We hypothesized that when released from the substrate (Figure 1b), FN within the fabrics transitions from the extended conformation to a more compact conformation. To test this hypothesis, we measured the Raman spectra for seven FN fabrics before and after release from the substrate. When adhered to the PIPAAm substrate, signature amide I $(1628,1651,1659$ $\left.\mathrm{cm}^{-1}\right)$, amide II $\left(1557 \mathrm{~cm}^{-1}\right)$, and amide III $\left(1266,1279,1283 \mathrm{~cm}^{-1}\right)$ peaks, attributed to the protein's secondary structure, are visible (Figure 1c, Supporting Information Figure S1e). ${ }^{20-21}$ When the nanoFabrics are released from the surface, only the amide I peak $\left(1659 \mathrm{~cm}^{-1}\right)$ is visible (Figure 1c). The loss in secondary structure peaks in the Raman spectra suggests that when FN is released from the substrate, it refolds from an extended conformation to a globular configuration, obscuring the amide II and III regions.

In order to investigate strain dependant changes in FN structure during release of the fabric from the substrate, we labeled FN with donor fluorophores on the primary amines and acceptor 
fluorophores on the free sulfhydryls of the $\mathrm{FN} \mathrm{III}_{7}$ and $\mathrm{FN} \mathrm{III}_{15}$ domains in a manner similar to previous reports. ${ }^{1,17-18,22-23}$ We measured the temporal changes in fluorescence resonance energy transfer (FRET) intensity before, during, and after the labeled fabrics were released from the PIPAAm substrate (Figure 2a). We observed an exponential increase in the ratio between the acceptor and donor emission intensities $\left(I_{A} / I_{D}\right)$ until fabrics were freed from the PIPAAm substrate and contraction was completed ( $\mathrm{t}=560 \mathrm{sec}$, Figure $2 \mathrm{~b}$ ). When the labeled FN is constrained to the PIPAAm surface (Figure 2c), FN is in an extended conformation, with minimal FRET signal (Figure 2d). As the PIPAAm substrate dissolves (Figure 2e), FRET fluorescence increases by $90 \pm 1 \%$, suggesting that $\mathrm{FN}$ has folded back to a globular form when the fabric is released (Figure 2f). To confirm that FRET in the contracting fabrics is due to FN conformational changes rather than the increasing proximity of donors and acceptors on adjacent FN molecules, fabrics containing an even mixture of FN labeled with only donor fluorophores and FN labeled with only acceptor fluorophores were released from a PIPAAm substrate (Supporting Information Figure S2). We observed only a $30 \pm 8 \%$ increase in FRET signal associated with intermolecular FRET signal (Supporting Information Figure S2a and Sc), indicating that the significantly higher increase in FRET fluorescence within the dual-labeled FN fabrics is due to intramolecular conformational changes. Both the enhanced FRET signal associated with FN contraction and the change in Raman signal suggest that FN within the released fabrics is in a compact conformation, while FN in patterned fabrics constrained to the substrate is in an extended conformation.

We asked how the secondary structural changes of FN affect the bulk mechanical properties of the released FN fabrics. To measure the mechanical properties of the fabrics, they were attached to calibrated glass microneedles via non-specific adhesive forces and uniaxially loaded 
at a constant rate of $1 \mu \mathrm{m} \mathrm{sec}^{-1}$ (Figure 3a). During uniaxial, tensile loading, the stretched fabric exhibited three regimes: a linear elastic regime, a stress plateau, and a strain stiffening regime (Figure 3b). This data suggests that relaxed fabrics undergo multiple transition points under mechanical strain.

To determine the molecular mechanism of fabric elongation, we used an eight-chain model to analyze the measured stretch-stress data and infer how FN within the fabrics responds during stretching. The eight-chain model is a well developed model that connects continuum level mechanics to the micro-structural level force-extension behavior. ${ }^{24}$ It was originally developed to describe the behavior of rubber and polymeric materials ${ }^{24}$ but has recently been applied to understand the contribution of protein unfolding in fibrin protein gels. ${ }^{25}$ The model idealizes a local, random network structure as semi-flexible chains connected at the center of a cube (Figure 3c). A flow diagram of the interaction of the model and experimental data is depicted in Supplementary Figure S3 in the Supporting Information. Here, each semi-flexible chain represents a single FN molecule. Using the model, the nominal stress $(N)$-stretch $(\lambda)$ relation of the fabric can be easily expressed in terms of force $(F)$ and extension $\left(\lambda_{c}\right)$ for the individual FN molecules (Figure 3d).

$$
N=\left(\lambda-\frac{1}{\lambda^{2}}\right) \frac{v r_{o}}{3 \lambda_{c}} F\left(\lambda_{c}\right)
$$

where

$$
\lambda_{c}=\sqrt{\left(\lambda^{2}+2 / \lambda\right) / 3}
$$


and $r_{o}$ and $v$ are the end-to-end length of the unloaded FN molecule and FN density within the cube, respectively. Using our experimentally measured stress $(N)$-stretch $(\lambda)$ data (Figure 3b), we can solve eq 1 and eq 2 for $F\left(\lambda_{c}\right)$ and $\lambda_{c}$ to infer the average force-extension behavior of FN during fabric stretching (Figure 3d). Note that the single free parameter here is $r_{o}$ within the undeformed fabrics. Assuming that FN molecules within released fabrics are in the compact conformation $\left(r_{o}=15.5 \mathrm{~nm}^{26}\right)$, the solved average force-extension relation for FN exhibits a force plateau at $\sim 80 \mathrm{pN}$ (blue axis, Figure $3 \mathrm{~d}$ ). However, if we assume that $\mathrm{FN}$ is in an extended conformation within the unloaded fabrics $\left(r_{o}=160 \mathrm{~nm}^{11,27}\right)$, an extreme value of the force plateau $(\sim 8.6 \mathrm{nN})$ is predicted by the model (red axis, Figure $3 \mathrm{~d})$.

We used a two-state model to predict the force at which individual domains within FN begin to unfold. $^{12,25,28}$ Indeed, the force plateau observed at $80 \mathrm{pN}$ is well captured using the two-state model, indicating that FN III domains unfold (solid line, Figure 3d). This observation is consistent with a study that suggests FN III domain unfolding occurs between $80-130 \mathrm{pN}$ during single molecule force spectroscopy (see Supporting Material). ${ }^{12}$ Results from the twostate model are combined with the eight-chain network model to predict the fabric stress-stretch data (solid line, Figure 3d) with good agreement to the experimental results. However, if FN fabrics are modeled as a two-state model with a network of non-interacting molecules aligned in the direction of the applied stretch, the model prediction largely underestimates the experimental results (dashed line, Figure 3b). These data suggest that FN is polymerized as a 3D network of connected molecules within the released fabrics. Furthermore, the combined two-state and eightchain network models predict that the transition points observed within the deformed fabric are due to conformational changes within the linear elastic regime $(\lambda<2)$, a threshold value where, when exceeded, domain unfolding occurs $(\lambda>2)$. 
We asked how conformational extension and domain unfolding contribute to the elasticity and plasticity of FN fabrics. Four fabrics are stretched beyond the domain unfolding regime $(\lambda=$ 4-5.5), after which point they are slowly $\left(1 \mu \mathrm{m} \mathrm{sec}^{-1}\right)$ unloaded (Figure 4a, Supporting

Information Figure S4). The length recovery kinetics of the pre-stretched fabrics under zero load is measured (Figure 4b, plotted in Figure 4c) and plotted as a function of recovery time (circles, Figure 4c and Supporting Information Figure S4a-c). Data is fit to a double exponential curve (solid line, Figure 4c and Supporting Information Figure S4a-c) with $\lambda=A_{1} e^{\frac{-t}{\tau_{1}}}+A_{2} e^{\frac{-t}{\tau_{2}}}$. The variables $A_{1}$ and $A_{2}$ represent the decay amplitude and fit parameter, respectively; $\tau_{1}$ and $\tau_{2}$ represent the half lifetimes for fast $(81 \pm 11 \mathrm{sec})$ and slow $\left(19 \pm 9 \mathrm{E}^{4} \mathrm{sec}\right)$ recovery, respectively, and are delineated in Figure 4c and Supporting Information Figure S4a-c by the vertical dashed line. In spite of a $1400 \mathrm{sec}$ relaxation period, fabrics do not fully recover their original length, exhibiting a total permanent deformation $\left(\lambda_{\text {final }}-1\right)$ of $0.5 \pm 0.2$ (Figure $4 c$, Supporting Information Figure S4a-c). When the relaxed fabrics are stretched again, they exhibit altered mechanical properties, displaying an increase in stretch-stiffening (Figure 4d, Supporting Information Figure S4d-f). The change in mechanical behavior as a function of maximum load is known as the Mullins effect, which is caused by damage or debonding within single polymer chains. $^{29-31}$ Our data suggests that large strains may damage the original configuration of the FN III domains, reminiscent of a Mullins effect, and that this damage irreversibly alters the length and mechanical properties of the FN fabrics.

To determine the threshold of plastic deformation, we cyclically loaded three fabrics, gradually increasing the maximum applied stretch from $\lambda_{\max }=1.5$ up to $\lambda_{\max }=6.0$ over 11 cycles at a $1 \mu \mathrm{m} \mathrm{sec}^{-1}$. The stress-stretch response in each cycle (Figure 4e, Supporting Information Figure S5) and the total permanent deformation (Figure 4e, inset, Supporting Information Figure 
$\mathrm{S} 5$, insets) were measured as a function of the maximum applied stretch. As the fabrics are cyclically loaded, they are allowed to relax for $500 \mathrm{sec}$, which is the duration of the fast recovery time deduced from the length recovery kinetics (Figure 4c, Supporting Information Figure S4ac). Plastic deformation is initiated by $\lambda=2$, at which point the fabrics are no longer able to recover their original length. Rate dependence on the plasticity was not investigated; thus, a viscoplastic response is not detected. Our results suggest that once the fabrics are stretched beyond this threshold, domain unfolding occurs, causing the permanent deformation of FN in the fabrics. Both the experimental and numerical results demonstrate that FN elasticity within fabrics is due to conformational changes under low strains $(\lambda<2)$, and that at high strains $(\lambda>2)$, domain unfolding potentiates plastic deformations.

Until now, the molecular mechanism accounting for FN elasticity has been debated between two structural models. In the first model, elasticity within FN fibers is due to conformational extension of polymerized FN without domain unfolding. ${ }^{10,16}$ In the second model, relaxed FN matrix fibers contain FN molecules that are already in an extended conformation, and elasticity is due to individual FN III domains that unfold and refold within the fibril. ${ }^{17-18}$ We report results in support of the first model and found that FN elastically deforms at low extensions $(\lambda<2)$ due to conformational unfolding. Beyond this threshold, FN plastically deforms due to domain unfolding.

A recent report suggested that FN nanofibers formed from a droplet exhibited a complete elastic recoil, even after large extensions $(\lambda=3.5) ;{ }^{22}$ however, we argue that this observation is dependent on the fabrication method. Due to the nature of their synthesis, these fibers pulled from a droplet ${ }^{22}$ experience a pre-stretch of $\lambda=2.4$, as they are deposited prior to mechanical testing. According to our molecular model and experimental data, the pre-stretch initiated during 
deposition is enough to induce a plastic deformation on the pulled FN fibers prior to any mechanical testing. We argue that once the fiber has crossed the plastic deformation threshold, its mechanical properties are permanently altered and this may account for its ability to recover elastically after large strains.

Models attempting to explain the mechanism for FN unfolding have yet to fully recapitulate experimental stress-strain curves. For example, FN modeled as wormlike-chain molecules linked in a one-dimensional (1D) series produces a much narrower strain regime for molecular unfolding, than is observed experimentally. ${ }^{32}$ The authors reported an improved network model where FN is organized in parallel, but the model does not fully capture the isotropic mechanical property of the stress-free network. ${ }^{32}$ The micro-structurally informed eight-chain network and two-state relation used in our manuscript, correct for this short-coming with good agreement to the experimentally observed unfolding, indicating that 3D network models successfully recapitulate the bulk mechanical properties of a FN network.

In conclusion, we have identified how protein textiles composed of polymerized FN respond to mechanical load across multiple spatial scales, from the network down to the molecular level. Fabrics are capable of extending up to nine times their original length without breaking, making them one of the most extensible engineered protein networks. Our experimental findings support a mechanism for FN extensibility that occurs in sequential stages activated by elastic deformation at $\lambda<2$, plastic deformation at $2<\lambda<5$, and stretch stiffening at $\lambda>5$. We observe that the average force associated with plastic deformation within the loaded fabrics at the $\lambda=2$ threshold is $131 \pm 17 \mathrm{nN}$ ( $\mathrm{n}=5$ fibers). Single cells can generate forces between $20-70 \mathrm{nN} .{ }^{2,4}$ Thus, we reason that cell-generated forces are not large enough to trigger domain unfolding and plastic deformation on their ECM, which may explain why the ECM is 
elastic in vivo. Having used FN as a model protein, we can now use the eight-chain and two-state models as a means to predict how molecular structure, elasticity, and extensibility are altered in the presence of mechanical load within other natural or synthetic multimodular proteins. Protein analogues embedded with domains similar in structure and function to the FN III domains of FN may offer promising substrates for failure-proof sealants or hyperelastic textiles. 

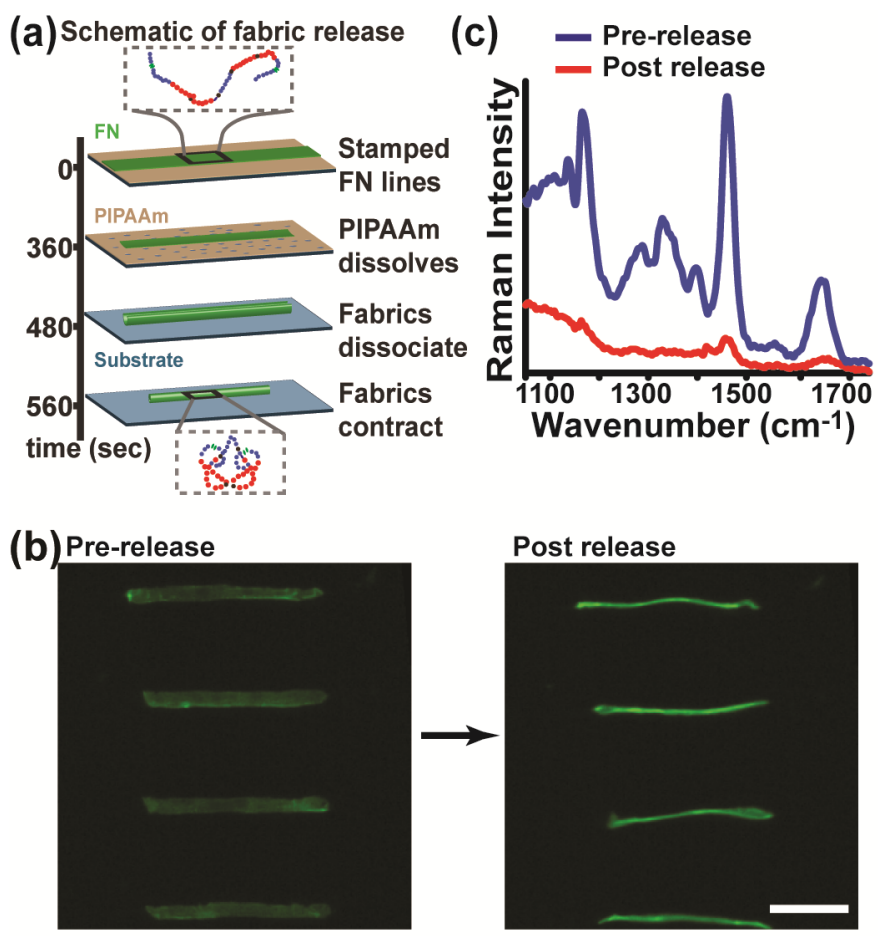

Figure 1. Forming FN textiles. (a) Schematic of conformational changes in FN. FN is extended under tension as micropatterned ribbons. Once the PIPAAM substrate dissolves, tension is released, and FN contracts into compact conformation forming fabrics. (b) As PIPAAm dissolves, fluorescently labeled FN nanoFabrics fold into free-standing fibrillar arrays. Scale bar is $50 \mu \mathrm{m}$. (c) Raman spectra of FN Fabrics pre- (blue) and post- (red) release from the PIPAAm substrate indicate loss in the secondary structure of FN, once the fabrics contract. 
(a) Releasing fabrics

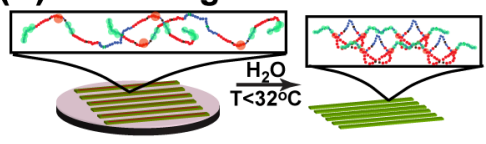

(c) Pre-release

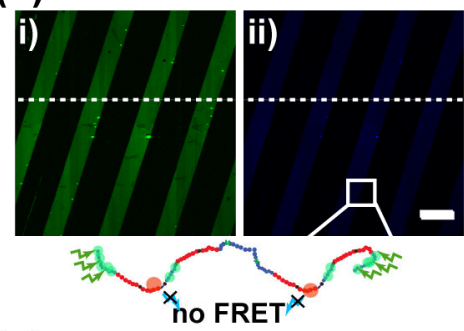

(e) Post-release

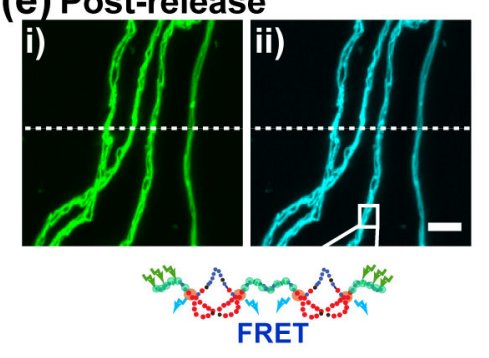

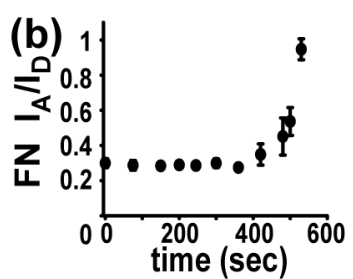

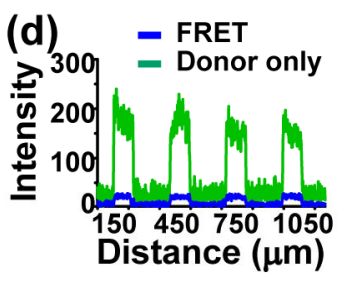

(f) = FRET

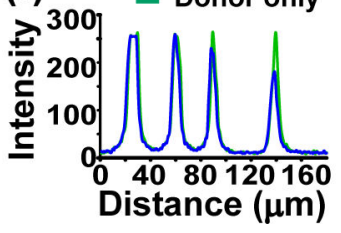

Figure 2. Optical signature of conformational changes in FN fabrics under strain. (a) Schematic of conformational changes within fabrics labeled for intramolecular FRET. (b) The ratio of peak acceptor to donor intensities $\left(\mathrm{I}_{\mathrm{A}} / \mathrm{I}_{\mathrm{D}}\right)$ of the releasing FRET-labeled FN plotted as a function of the time $(\mathrm{n}=8)$ indicates that released $\mathrm{FN}$ is in a compact conformation. (c) Unreleased FN ribbons excited at donor excitation wavelength $(488 \mathrm{~nm})$ were captured at donor emission wavelength $(522 \mathrm{~nm}, \mathrm{i})$ and acceptor emission wavelength $(576 \mathrm{~nm}, \mathrm{ii})$. (d) Intensity profiles taken along dotted line from scan (c) for both donor (green) and acceptor (blue) emission signal. (e) Released FN fabrics excited at donor excitation wavelength $(488 \mathrm{~nm})$ were captured at donor emission wavelength $(520 \mathrm{~nm}, \mathrm{i})$ and acceptor emission wavelength $(576 \mathrm{~nm}$, ii). (f) Intensity profiles taken along dotted line from scan (e) were plotted for both acceptor (green) and donor (blue) emission signal. Scale of c and e is $50 \mu \mathrm{m}$. Error bars in (b) indicate standard deviation. 

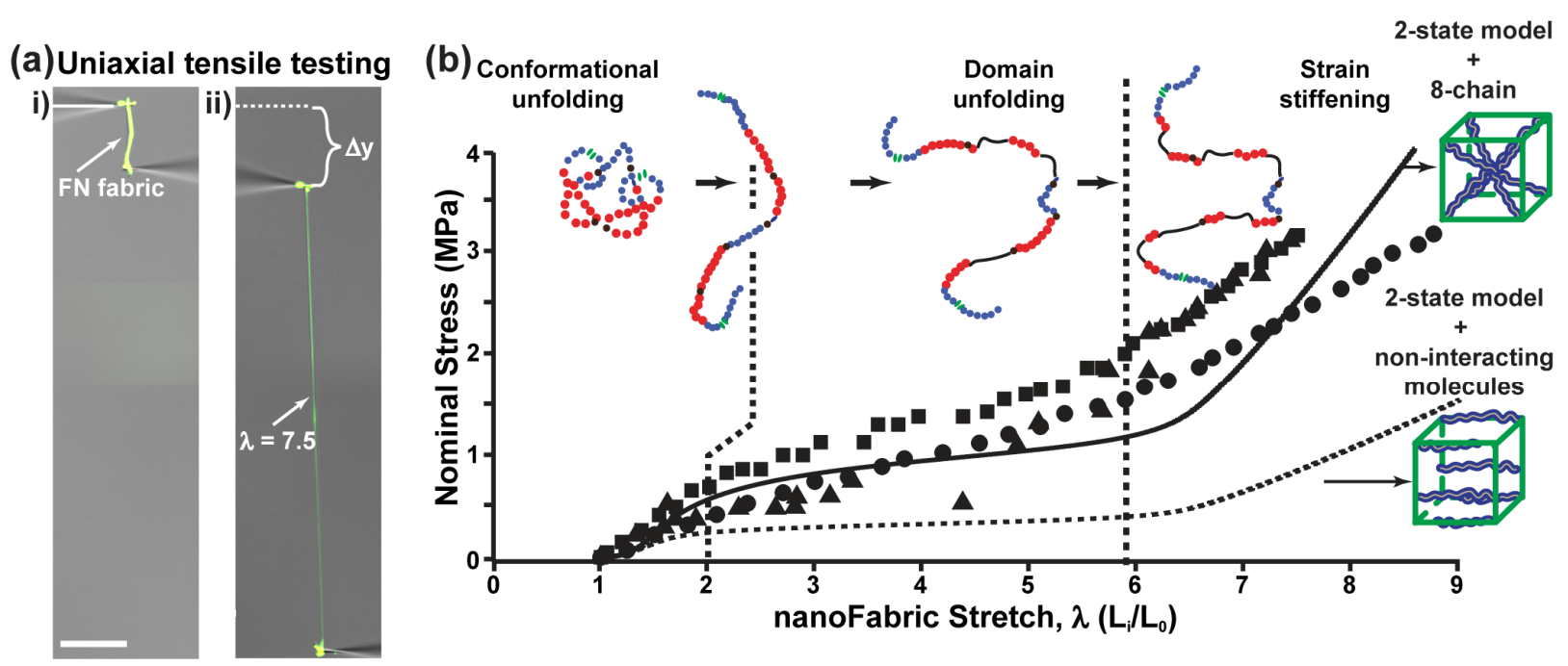

(c) Schematic of eight-chain model
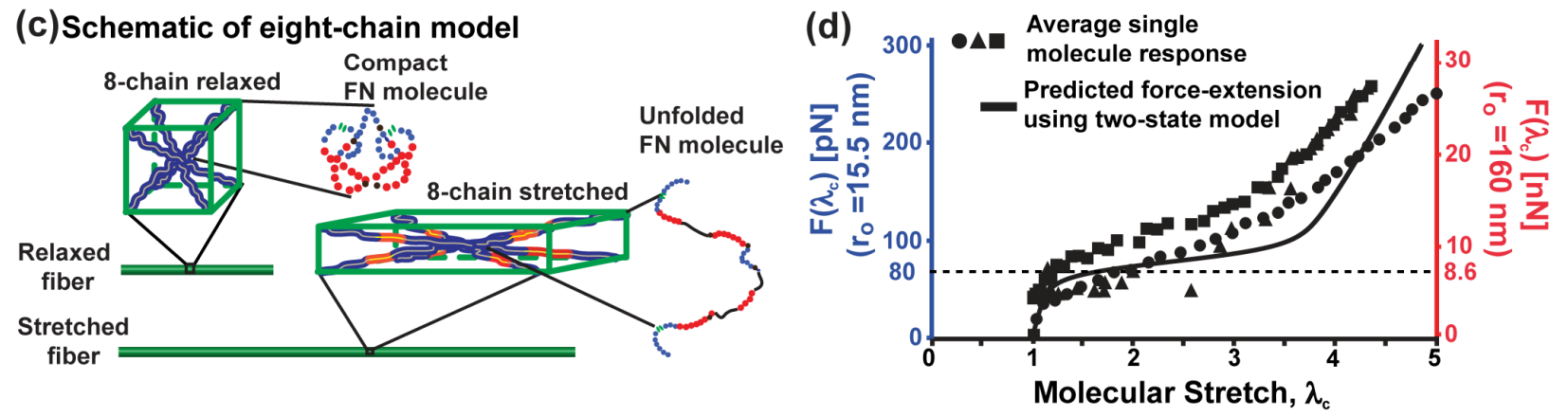

Figure 3. FN network extensibility modeled using the eight-chain and two-state models. (a) DIC and fluorescent merged images of a representative fabric (green) suspended at two ends by calibrated microneedles prior to uniaxial tensile testing (i). Deflection $(\Delta y)$ at the tip-fiber interface represents load generated during extension (ii). Scale bar is $100 \mu \mathrm{m}$. (b) Stress-stretch curves for loaded nanoFabrics ( $\mathrm{n}=3$, squares, triangles, and circles) at a constant rate $\left(1 \mu \mathrm{m} \mathrm{sec}^{-}\right.$ ${ }^{1}$ ). The solid line represents the numerical results of the stress-stretch relation as predicted single molecule mechanics, the two-state model, and eight-chain network model. The dashed line represents if the FN network is modeled as a two-state system composed of non-interacting molecules. (c) Schematic of the eight-chain model to describe FN network assembly. The FN network is modeled as cubes with eight FN molecules (chains) connected at the centers. Under 
tensile load, the cubes deform. When the stretching force is large enough, $\beta$-sheets within FNIII domains begin to unfold (represented in orange in the stretched chains). (d) Average forceextension relation of a single FN molecule estimated using the eight-chain model ( $\mathrm{n}=3$, squares, triangles, and circles). The solid line represents the numerical results of the force-extension relation as predicted by the two-state model. Blue axis is force $(\mathrm{pN})$ assuming reference length of FN within an unloaded fabric is compact, with $r_{o}$ of $15.5 \mathrm{~nm}$. The red axis is force $(\mathrm{nN})$ assuming the reference length of FN within an unloaded fabrics is extended, with $r_{o}$ of $160 \mathrm{~nm}$. Dashed horizontal line depicts the force threshold obtained the different $r_{o}$ values indicated on the vertical axes. 

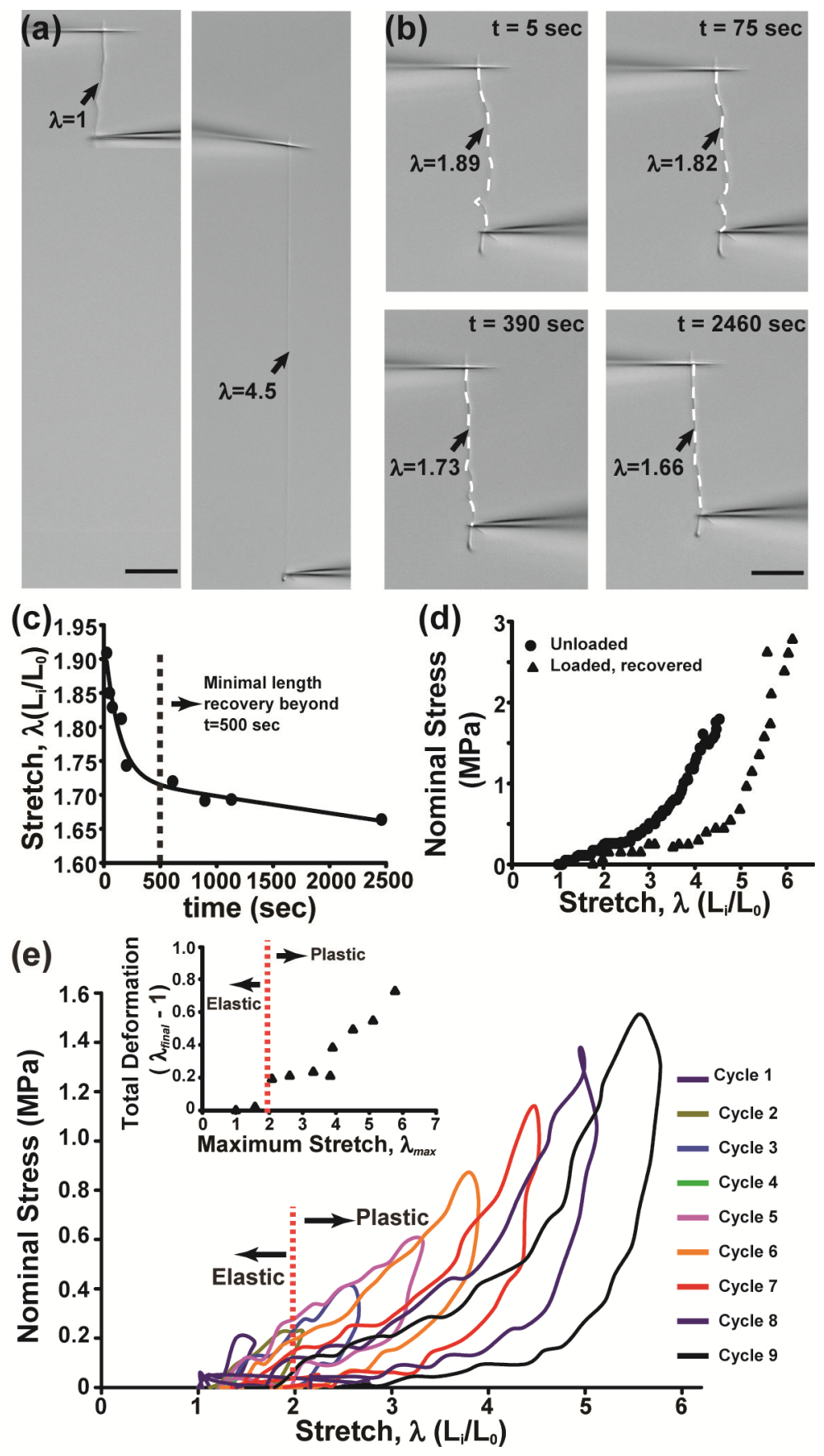

Figure 4: Length recovery dynamics of stretched fabrics indicate threshold for plastic deformation at $\lambda=2$. (a) An unloaded fabric $(\lambda=1)$ is stretched to $\lambda=4.5$ at a constant rate of 1 $\mu \mathrm{m} \mathrm{sec}{ }^{-1}$. (b) The stretched fabric is manually unloaded, and its length recovery is optically measured over $2460 \mathrm{sec}$. (c) The recovered stretch was plotted as a function of recovery time 
(circles). (d) Stress-stretch curves were measured for the unloaded fabrics before stretch (circles) and after loading and recovery (triangles). (e) Stress-stretch curves of a cyclically loaded fabric. The total permanent stretch on the fabrics is calculated (inset) by normalizing the final stretch $\left(\lambda_{\text {final }}\right)$ to the original, unloaded fabric $\left(L_{o}=1\right)$. The red dotted line indicates threshold for plastic deformation in both graphs. Scale of A and B is $50 \mu \mathrm{m}$. 


\section{TOC:}

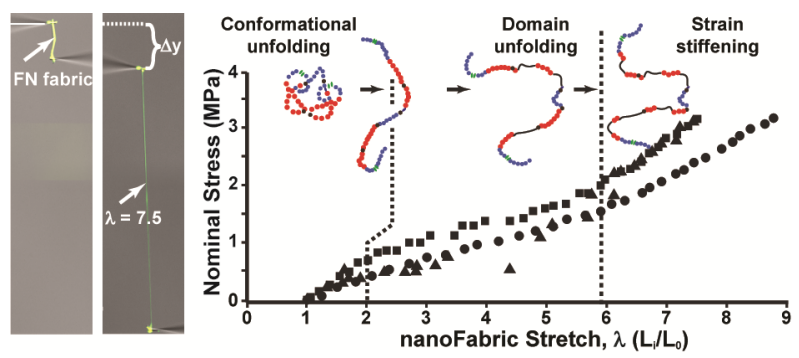

Supporting Information. Experimental methods and models are described in detail in the online supplement. This material is available free of charge via the Internet at http://pubs.acs.org.

\section{Corresponding Author}

* Kevin Kit Parker

Harvard School of Engineering and Applied Sciences

29 Oxford St (Rm 321)

Cambridge, MA 02138

Phone: (617) 495-2850

Fax: (617) 496-1793

E-mail: kkparker@seas.harvard.edu

\section{Author Contributions}

The manuscript was written through contributions of all authors. All authors have given approval to the final version of the manuscript.

\section{Funding Sources}

This work was partially funded by the Defense Advanced Research Projects Agency CINAPSE (W911NF-10-1-0113 for K.K.P.), Harvard University Nanoscale Science and Engineering Center supported by the National Science Foundation (PHY-0646094 for K.K.P.), Harvard University Materials Research Science and Engineering Center supported by the National 
Science Foundation (DMR-0213805 of K.K.P. and K.B.), and a Draper Lab Fellowship (J.W.R.). K.B. acknowledges startup funds from the School of Engineering and Applied Sciences, Harvard and the support of the Kavli Institute at Harvard University

The authors wish to thank Professor Patrick Alford, Professor Anna Grosberg, and Mr. Borna Dabiri for their comments on the manuscript. The authors gratefully acknowledge the use of facilities at the Harvard Center for Nanoscale Systems and the Wyss Institute for Biologically Inspired Engineering. 
1. Antia, M.; Baneyx, G.; Kubow, K. E.; Vogel, V. Faraday Discussions 2008, 139, 229249.

2. $\quad$ Balaban, N. Q.; Schwarz, U. S.; Riveline, D.; Goichberg, P.; Tzur, G.; Sabanay, I.; Mahalu, D.; Safran, S.; Bershadsky, A.; Addadi, L.; Geiger, B. Nature Cell Biology 2001, 3, (5), 466-472.

3. Chiquet, M.; Reneda, A. S.; Huber, F.; Fluck, M. Matrix Biology 2003, 22, (1), 73-80.

4. $\quad$ Geiger, B.; Bershadsky, A.; Pankov, R.; Yamada, K. M. Nature Reviews Molecular Cell Biology 2001, 2, (11), 793-805.

5. Ingber, D. E. Circulation Research 2002, 91, (10), 877-887.

6. Sottile, J.; Hocking, D. C. Molecular Biology of the Cell 2002, 13, (10), 3546-3559.

7. Vogel, V., Mechanotransduction involving multimodular proteins: Converting force into biochemical signals. In Annual Review of Biophysics and Biomolecular Structure, 2006; Vol. 35, pp 459-488.

8. Branden, C.; Tooze, J., Introduction to protein structure. 2nd ed.; Garland Publishing, Inc: New York, 1991.

9. Heim, M.; Romer, L.; Scheibel, T. Chemical Society Reviews 2010, 39, (1), 156-164.

10. Erickson, H. P. Journal of Muscle Research and Cell Motility 2002, 23, (5-6), 575-580.

11. Mao, Y.; Schwarzbauer, J. E. Matrix Biology 2005, 24, (6), 389-399.

12. Oberdorfer, Y.; Fuchs, H.; Janshoff, A. Langmuir 2000, 16, (26), 9955-9958.

13. Schwarzbauer, J. E.; Sechler, J. L. Current Opinion in Cell Biology 1999, 11, (5), 622-

627.

14. Wierzbicka-Patynowski, I.; Schwarzbauer, J. E. Journal of Cell Science 2003, 116, (16), 3269-3276.

15. Gee, E. P. S.; Ingber, D. E.; Stultz, C. M. Plos One 2008, 3, (6).

16. Abu-Lail, N. I.; Ohashi, T.; Clark, R. L.; Erickson, H. P.; Zauscher, S. Matrix Biology 2006, 25, (3), 175-184.

17. Baneyx, G.; Baugh, L.; Vogel, V. Proceedings of the National Academy of Sciences of the United States of America 2001, 98, (25), 14464-14468.

18. Baneyx, G.; Baugh, L.; Vogel, V. Proceedings of the National Academy of Sciences of the United States of America 2002, 99, (8), 5139-5143.

19. Feinberg, A. W.; Parker, K. K. Nano Letters 2010, 10, (6), 2184-2191.

20. Baujard-Lamotte, L.; Noinville, S.; Goubard, F.; Marque, P.; Pauthe, E. Colloids and Surfaces B-Biointerfaces 2008, 63, (1), 129-137.

21. Strehle, M. A.; Rosch, P.; Petry, R.; Hauck, A.; Thull, R.; Kiefer, W.; Popp, J. Physical Chemistry Chemical Physics 2004, 6, (22), 5232-5236.

22. Klotzsch, E.; Smith, M. L.; Kubow, K. E.; Muntwyler, S.; Little, W. C.; Beyeler, F.; Gourdon, D.; Nelson, B. J.; Vogel, V. Proceedings of the National Academy of Sciences of the United States of America 2009, 106, (43), 18267-18272.

23. Little, W. C.; Smith, M. L.; Ebneter, U.; Vogel, V. Matrix Biology 2008, 27, (5), 451461.

24. Arruda, E. M.; Boyce, M. C. Journal of the Mechanics and Physics of Solids 1993, 41, (2), 389-412.

25. Brown, A. E. X.; Litvinov, R. I.; Discher, D. E.; Purohit, P. K.; Weisel, J. W. Science 2009, 325, (5941), 741-744. 
26. Koteliansky, V. E.; Glukhova, M. A.; Bejanian, M. V.; Smirnov, V. N.; Filimonov, V. V.; Zalite, O. M.; Venyaminov, S. Y. European Journal of Biochemistry 1981, 119, (3), 619624.

27. Erickson, H. P.; Carrell, N. A. Journal of Biological Chemistry 1983, 258, (23), 45394544 .

28. Bell, G. I. Science 1978, 200, (4342), 618-627.

29. Mullins, L. Rubber Chem. Technol 1969, 42, 339-362.

30. Ogden, R. W.; Roxburgh, D. G. Proceedings of the Royal Society of London Series aMathematical Physical and Engineering Sciences 1999, 455, (1988), 2861-2877.

31. Horgan, C. O.; Saccomandi, G. Mathematics and Mechanics of Solids 2002, 7, (4), 353371.

32. Bradshaw, M. J.; Smith, M. L. Biophysical Journal 2011, 101, (7), 1740-1748. 